\title{
FIGURE AND FINISH OF GRAZING INCIDENCE MIRRORS*
}

Peter Z. TAKACS

Brookhaven National Laboratory, Upton, NY 11973

and

Eugene L. CHURCH

U. S. Amy ARDEC, Picatinny, NJ 07801
BNL -42887

DE9 $0 \quad 012872$

\section{Abstract}

Great improvement has been made in the past several years in the quality of optical components used in synchrotron radiation (SR) beamlines. Most of inis progress has been the result of vastly improved metrology techniques and instrumentation permitting rapid and accurate measurement of the surface finish and figure on grazing incidence optics. A significant theoretical effort has linked the actual performance of components used at $\mathrm{x}$-ray wavelengths to their topological properties as measured by surface profiling instruments. Next-generation advanced light sources will require optical components and systems to have sub-arc second surface figure tolerances. We will explore the consequences of these requirements in terms of manufacturing tolerances to see if the present manufacturing stateof-the-art is capable of producing the required surfaces.

It is now possible to routinely measure figure, and finish on meter-long optical surfaces in our laboratory with a long trace profiler of our own design and with a commercial microinterferometer profiler. Our instrumentation allows us to analyze surface profile data in terms of the power spectral density function over the range of spatial periods

"This work was supported by the U. S. Department of Energy: Contract No. DE-AC02-76CH00016. 
that are of relevance to $\mathrm{x}$-ray mirror performance: $1 \mathrm{~m}$ to $5 \mu \mathrm{m}$. We have been able to recognize several classes of problems in the manufacture of grazing incidence optics from the results of our measurements. In some cases we have been able to provide feedback to the manufacturer, who has then been able to modify and improve his process. Results from our analyses of mirror figure and finish measurements will be presented and their impact on the design of high-resolution soft $x$-ray SR systems will be discussed.

\section{Introduction}

The manufacture of $\mathrm{x}$-ray mirrors is a rather specialized branch of the optical fabrication industry. As those who have had to deal with the procurement of these components well know, there are only a handful of optical companies who supply most of the grazing incidence optics in use at the synchrotron light source facilities around the world. The reasons for this are several. Firstly, production of a grazing incidence asphere is usually a one-of-a-kind job. It does not lend itself to mass production methods. Not many optical houses are willing to commit the necessary resources for the fabrication of one-of-a-kind components. Secondly, because it is a one-of-a-kind job, it is relatively laborintensive, which drives up the cost on each individual item. There is a great deal of engineering and planning that must go into the fixturing and design of the computer control program for numerically-controlled diamond turning machines for production of metal optics. Most end users tend to view the procurement of aspheric x-ray optics as a catalog process, i.e., that all one needs to do is choose the desired parameters from the manufacturers catalog of stock items and then expect delivery of the finished item (after considerable delay beyond the initial delivery date) which will then perform flawlessly as desired. In reality the procurement process is quite complex, starting with the initial trade- 
off studies to choose between metal optics or glass-ceramic optics, continuing with the selection of a vendor who can best do the job, monitoring the progress of the job and ending with the final quality assurance acceptance inspection. Many times the component fails to pass the inspection and is returncd for further rework. There is relatively little information available of practical use to guide the user through any of the above steps. We have been forced to develop our own foundation for assessing the performance of various vendors and determining the quality of the components produced by them. Our approach has been to concentrate on the area of metrology of grazing incidence optics and to develop instruments and techniques that can be used to improve the quality of components delivered to us. The major problem hindering the production of grazing incidence optics is the lack of specialized metrology instrumentation that can be used by the small manufacturing shop to assess the quality of the component under production. We have been engaged over the past several years in developing the theoretical framework and practical measurement techniques to link the metrology to actual performance, providing much-needed feedback to the manufacturer and also educating users and manufacturers in the proper understanding of the language of surface figure and finish metrology.

\section{Theoretical Foundation}

In order to assess the performance of x-ray mirrors in a meaningful way, we must first decide what it is that we need to measure. Certainly we would like to know what the image quality is for light reflected from the mirror surface at the wavelength of operation-nominally in the 1 to $10 \AA$ range. To do this test at the operating wavelength would be very time-consuming and would require a very elaborate source and experimental chamber which could accommodate various types and sizes of mirrors with assorted focal 
parameters. So we are led to ask-"What is it that we can measure on grazing incidence optics?" We would like to measure mirrors off-line, in the laboratory, at visible wavelengths. We would like to measure those characteristics of the surface with visible light that affect the performance of the mirror at $x$-ray wavelengths. The parameters of the surface that meet these criteria are mirror figure and finish. We can measure the surface roughness on a microscopic scale and relate the measured quantities to the amount of light scattered from the surface, and we can measure the gross figure errors and relate them to degradation of the image quality.

The terms figure and finish are rather vague and do not lend themselves to a good quantitative definition. Historically, the division between figure and finish is based upon the types of instrumentation available to make each measurement-conventional interferometry and fringe pattern analysis for figure measurement and visible lightscattering measurements for finish. These methods are totally unsuited for metrology of $\mathrm{x}$-ray optics because conventional interferometry fails at measuring aspheric optics and visible light-scattering probes the surface at spatial periods that are irrelevant to the cause of scattering at $\mathrm{x}$-ray wavelengths. The region of surface spatial periods that is most important, from an x-ray mirror standpoint, lies between the regions probed by the two conventional methods. Fortunately, over the past few years, commercial instrumentation has been developed that allows one to easily measure the surface finish over the range of spatial periods that is appropriate to near-angle x-ray scattering at grazing incidence [1-4]. These are the spatial periods from several millimeters down to several microns in length. We have employed a microinterferometer system since 1984 to measure surface profiles over a $5 \mathrm{~mm}$ trace length with better than $1 \AA$ RMS repeatability [5]. This non-contact method of measuring the surface profile has made it very easy to quantify the quality of 
full-scale optical components in our laboratory. We have not been so fortunate in the area of figure me:surement. No commercial instrument exists that is versatile enough to perform metrology inspections on full-size aspheric optics with a minimum of set-up time. We have been forced to develop our own instrumentation for this task.

But it is not enough to just be able to measure the profile of a surface, one must relate the profile measurements to the desired functional parameters of the mirror under actual operating conditions which are quite different from the normal incidence, visible wavelength metrology conditions. We have devoted considerable theoretical effort over the past several years to the development of the tools necessary to make the connection between surface profile measurements and actual performance at $x$-ray wavelengths $[6,7]$. Perhaps the most significant result of our research has been the successful application of the measured surface power spectral density (PSD) function to the prediction of nearangle scattering of $x$-rays from a smooth mirror surface [8]. The surface PSD function is generated by computing the Fourier transform of individual surface profile measurements and then averaging all Fourier transforms for a given surface. We have shown that the Rayleigh-Rice form of the angle-resolved scattering equation at grazing incidence accurately describes the distribution and intensity of $x$-rays about the specular direction [7]. The scattering equation is:

$$
\frac{1}{I_{i}}\left[\frac{d I}{d \theta}\right]_{,}=(1-g) R\left(\theta_{i}\right) \delta\left(\theta_{s}-\theta_{i}\right)+16 \pi^{2}\left[\frac{\sin \theta_{i}}{\lambda}\right]^{3} \cdot R\left(\theta_{j}\right) \cdot K \cdot S(f)
$$

where $S(f)$ is the PSD curve as a function of surface spatial frequency, $f, R\left(\theta_{i}\right)$ is the Fresnel reflectivity curve at the grazing incidence angle $\theta_{i}$ and $K$ is the materials obliquity factor which depends on the particular theoretical approach used to generate the grazing 
incidence approximation. The Rayleigh-Rice formalism appears to provide the best fit to the measured scattering data. The form of $K$ for this case is relatively simple:

$$
K=\left[\begin{array}{l}
\theta_{s} \\
\bar{\theta}_{i}
\end{array}\right]^{2} \sqrt{\frac{R\left(\theta_{s}\right)}{R\left(\theta_{i}\right)}}
$$

The predictions of this theory have been borne out in a set of angle-resolved $x$-ray scatter measurements at $1.39 \AA$ wavelength from a smooth Au-coated SiC surface at several angles of incidence. Figure 1 illustrates a typical measured surface profile for this surface. The average PSD curve over ten random locations on the surface is shown in Fig. 2. The RMS roughness for this surface is about $3 \AA$ over the full bandwidth. The PSD curve is multiplied by the appropriate factors in Eq. (1) for each of the three angles of incidence and the scaled PSD curves are then plotted over the measured $x$-ray scattering curves in Fig. 3. The fit of the theoretical curves based on the PSD function to the measured $x$-ray scattering curves is quite good. The Rayleigh-Rice formalism accurately predicts both the shape and magnitude of the scattered light distribution and successfully predicts the asymmetry in the wings of the scattered light distribution, which is especially noticeable at the most grazing angle of incidence.

\section{Practical Considerations}

We have demonstrated that we can relate the scattered light distribution at $\mathrm{x}$-ray wavelengths to the surface roughness properties of a mirror surface through the appropriate theoretical framework. The question now is, "How do we apply this information to the procurement of useable grazing incidence optical components?" The answer to this question is not simple or easy. In the ideal world, if the user knew exactly what he needed 
in terms of the scattered light distribution from each surface, then it would be a relatively straightforward matter to work backwards through the equation to determine the maximum allowable shape and magnitude for the surface PSD curve. As a rule of thumb one can make a zero-order assumption that the PSD curves for glass-ceramics all have the same power-law shape, only the absolute magnitudes are different. The same holds true for the metal surfaces, although the power-law parameters are usually much different for them.

In practice, the user or optical system designer does not usually have a complete description of the optical system to allow him to deduce the required surface roughness spectrum. Only the most sophisticated of design codes include the surface PSD function as a design parameter [9]. Other factors which need to be considered are the type of detector that will be used (film, ion chamber, photon counter, etc.), whether or not it is an imaging system, what is the minimum required resolution, the required spectral purity for a monochromator system, the source size, the point spread function caused by figure errors, etc. All of these factors tend to complicate the specification of optical components. Contributing to the lack of rigor in grazing incidence system design has been that, in the past, it has not been possible to perform the required metrology on the mirrors. Mirror quality has been relatively poor and the credibility of the design process has been rather low because the systems have never performed close to their design goals. Designers and users have resorted to simplistic, back-of-the-envelope calculations to specify important surface roughness parameters. Seemingly insignificant changes in these parameters often have a large effect in cost of the final product. Our method of PSD function measurement and performance prediction puts the design process on a much more solid foundation. 


\section{Mirror Supplier Database}

Our measurements of surface roughness began in 1984 after acquisition of a WYKO NCP-1000 Digital Optical Profiler [1]. This instrument allows us to measure the surface roughness of full-sized synchrotron mirrors in a non-contact manner, quickly and efficiently. In the several years since, we have compiled a database of surface roughness as a function of manufacturer, material, and figure type. Recognizing that there is an acute lack of practical information available to the grazing incidence community regarding the above parameters, we have compiled our information in a way that should prove useful for future design considerations.

The mirrors in the database are divided into two distinct classes: flats and aspheres. Conspicuously absent from this database are spherical surfaces. The only spherical surfaces measured to date have been for diffraction gratings and the number of samples is extremely small, so they are not included in this compilation. The aspheric surfaces include cylinders, toroids and ellipsoids. As with the figure types, there are two major material classes: metal substrates and glass or ceramic substrates. The only metal material that is included in this database is electroless nickel plate (ENP), usually on an aluminum substrate. The reason for this is that, for synchrotron use, no other metal surface can be polished to the smoothness required of an $\mathrm{x}$-ray reflecting surface. The fact that ENP can also be diamondmachined is also an important reason for its use in SR mirror fabrication.

The list of manufacturers providing mirrors to the NSLS and other synchrotron facilities whose mirrors have been measured in our Metrology Laboratory is presented in Table I. A summary of measured mirrors arranged by figure and material type is given in Table II with the number of mirrors of each type identified by manufacturer. Of all the mirrors in this table, only the General Optics silicon flats are not real SR mirrors. These 
are single-crystal silicon wafers and are included to demonstrate the high-quality optical finish that can be applied to this material if one is careful. Conventional silicon wafers for semiconductor applications are usually an order of magnitude worse in surface roughness.

Table II also summarizes the number of mirrors of each type that have been measured on the BNL WYKO profiler. Please note that not all of these mirrors are for use at the NSLS: a significant fraction were sent here for measurement and then shipped elsewhere for use at other locations, in particular SSRL, SRC in Wisconsin and CHESS. Also, some mirrors are entered twice in the statistics, usually a result of measuring before and after a reworking process. If the mirror failed to meet the specifications, it was sent back to the manufacturer and after reworking was returned here for a second measurement. The most striking examples of reworking to be noted later are early fused silica cylinders made by Frank Cooke around 1981-82, prior to the use of the WYKO profiler for surface metrology. Measurements made on these mirrors in 1985 showed that they had a large amount of low frequency ripple, resulting in RMS roughness numbers that ranged from 25

to $85 \AA$. The low frequency ripple with spatial periods in the millimeter range is extremely difficult to detect by any other means. Frank Cooke subsequently changed his lapping process and, as a result, new cylinders and reworked old cylinders now routinely measure at or below the $5 \AA$ RMS level.

\section{Surface Finish Measurements}

The RMS roughness of each mirror in each column of Table II is plotted roughly as a function of time in the diagram in Figs. 4 through 7 . Each figure and material combination is plotted on a separate graph to better enable visual comparisons between various permutations of the parameters. The horizontal axis labelled "Volume ID Number" 
refers to the data file identification number in the WYKO profiler log book. It is basically a time series with Volume 1 starting in 1984 and Volume 5 starting in January 1988 covering the first half of 1988 . The data points refer to the date on which the mirror was measured in the laboratory, not necessarily to the date the mirror was manufactured. In fact, some mirrors that were manufactured many years ago have only recently bisen measured. Most measurements in this database were made with the $2.5 \mathrm{X}$ magnification objective on our WYKO instrument. Only a few of the early measurements were made with the $10 \mathrm{X}$ objective. The $2.5 \mathrm{X}$ objective is now used almost exclusively, since it provides significant information about the roughness in the important spatial period region from 1 to $5 \mathrm{~mm}$. This is the region that is most inaccessible to other metrology techniques and is what makes our measurement capability unique. The roughness numbers plotted are for the full $2.5 \mathrm{X}$ bandwidth which covers the range from $5.0 \mathrm{~mm}$ down to $9.8 \mu \mathrm{m}$.

Figure 4 summarizes the results for glass/ceramic aspheres. The three types of aspheres measured are cylinders, ellipsoids, and toroids. Most of the mirrors are made from fused silica; a few are ZERODUR, two are single-crystal silicon and two are CVD silicon carbide. Some are coated with a metal reflecting layer, some are uncoated. We almost never see evidence that a properly-applied thin metal coating changes the roughness properties of the surface, although occasionally a bad coating does slip in.

The majority of glass/ceramic aspheres have been right circular cylinder segments, primarily used for sagittal focusing of a horizontal fan of synchrotron radiation. The group of 5 cylinders located in a vertical column at "A" in Fig. 4 were manufactured a few years earlier by Frank Cooke. As mentioned above, they were only first measured at the time indicated in the graph. Several other Frank Cooke cylinders with RMS roughness values above $20 \AA$ were also manufactured in this early time frame, but were only recently 
measured. Those whose early manufacture date could be confirmed are indicated with an arrow pointing to earlier times. The major trend to note is the significant reduction in the average RMS roughness of the cylinders in more recent times. The early cylinders were usually always above $20 \AA$ RMS, while now it is unusual to see one that exceeds $10 \AA$ RMS. A dramatic example of the improvement in the polishing process based on feedback from the metrology is indicated by the data points marked with asterisks. An early fused silica mirror with a roughness of about $40 \AA$ RMS was reworked and measured in 1988 to have an RMS roughness slightly more than $3 \AA$. Another example of improved surface finish is the series of three measurements on a cylinder ar " $\mathrm{B}$ " connected by the straight lines. The initial measurement was $23.3 \AA$ RMS. Additional work on the surface produced a $25 \AA$ surface (not statistically different from the first measurement), but additional work and a process change produced a surface with a $7.8 \AA$ RMS finish. The mirror at $\mathrm{C}$ was originally made years ago, but after reworking its roughness level decreased from $83 \AA$ to $5.89 \AA$ RMS. A subsequent platinum coating was measured at $5.4 \AA$ RMS, again not statistically different from the uncoated surface. Occasionally, however, a new mirror slips through with a very rough surface, as indicated by the point labeled " $D$ " at about $70 \AA$ RMS in the upper right corner. This illustrates the usefulness in being able to make these kinds of measurements on a regular basis. Other products in this class from other vendors all lie below the $10 \AA$ level, which differs significantly from the metal mirror results.

The data for the metal aspheres is plotted in Fig. 5. One can see almost immediately that there appears to be a lower limit of about $10 \AA$ RMS to the best that can be achieved on this material, independent of figure. The best surface to date in the electroless nickel material is the most recent product from Optic-Electronic Corp., a cylinder with an RMS roughness of $8.45 \AA$, but whether or not this low value can be improved upon remains an 
open question. All the evidence indicates that it is very difficult to produce a surface below the $10 \AA$ level with ENP, while for glass/ceramic materials it is highly unusual to exceed the $10 \AA$ level. Pairs of data points in Fig. 5 also indicate the improvement in surface finish of a set of ellipsoidal mirrors obtained after reworking the mirrors. In each case the rework involved additional polishing on the surfaces. The pair of data points above the $100 \AA$ level are for a rather complex ellipscid designed to produce an 8 to 1 image size reduction [10]. The numerical machining control problem was extremely difficult to solve to produce the desired contours with 3 degrees of freedom in the machine motion. Additional polishing was not able to remove the low frequencies left in the surface. The set of open squares labeled " $A$ " indicate before and after measurements on two ENP ellipsoids that were coated with gold-electroplate. Again, no change in the roughness was evident.

More compelling evidence for the $10 \AA$ roughness barrier can be seen by comparing the glass/ceramic flats in Fig. 6 with the ENP flats in Fig. 7. The two silicon carbide flats above $10 \AA$ are really grating test samples and should not be included in the comparison. The only other flat above $10 \AA$ is a piece of float glass with the unusual property of being very smooth along the pull direction, but with a well-defined, large amplitude sinusoidal low-frequency period in the transverse direction. A vertical line connects the two directions on the graph. Most of these flats are intended to be used in mirror benders to produce focusing in the tangential direction and, in the case of specially-configured cross-section pieces, focusing in the sagittal direction also. The ENP flats in Fig. 7 again show a distinct cutoff at the $10 \AA$ level. There are none better than $8 \AA$. The only example, of degradation in surface roughness after coating is seen at the right-hand side of Fig. 7. A thick platinum coating was evaporated onto a $70 \mathrm{~cm}$ long ENP mirror and the surface roughness increased from $15 \AA$ before to $35 \AA$ after coating. 


\section{Surface Finish Discussion}

The division line at $10 \AA$ between the ENP and glass/ceramic materials appears to be independent of figure type. The same division is seen in the data from the aspheres as from the flats. In fact, the best finishes for each material appear to be independent of figure type. One can achieve the same quality finish on aspheres as on flats, so there should be no need to relax finish performance requirements for non-flat surfaces. Aspheric surfaces are, however, more difficult to produce than are flats, especially when one desires a multiaxis machine cut, as with toroids and ellipsoids. Cylinders can be easily manufactured both in glass/ceramic and metal materials because only one uniform radius needs to be generated in the surface. Higher-order aspheres can be made more easily in metals than in glass, and subsequently for less cost per piece, but the performance may suffer because of the limits on polishability. The electroless nickel plating process is subject to many factors that are usually beyond the control of the mirror fabricator. There are many variables that go into the ENP process that are not controlled in an ordinary industrial plating operation. Mirror manufacturers often report that they had to have the ENP coating stripped and replated several times before the final diamond-machined and polished surface met the specifications. These iterations tend to drive the price up on metal mirrors. Very few manufacturers can adequately characterize the quality of the ENP coating before polishing the piece. These considerations are non-existent for glass/ceramic materials, except for the case of silicon carbide. A layer of chemical vapor deposited (CVD) $\mathrm{SiC}$ is deposited on the surface of another substrate material and the CVD layer is then polished to the desired finish. Like the ENP process, the CVD process is also subject to many variables which are often difficult to control. A different set of problems 
arise in polishing the $\mathrm{SiC}$, but the end result could be the same: the need to redeposit or replate more material and polish again.

Each material has its advantages and its drawbacks. Metal mirrors allow designers more flexibility in terms of optical design by permitting complex surfaces to be machined, but at the present time the price is poor surface finish. Glass/ceramic flats are easily made, but their usefulness in imaging is not very great unless they are bent. Perhaps one will need to spend more effort in engineering the cross-sectional shape of a flat mirror and in the design of the bending mechanism to produce the desired tangential and sagittal curvatures from a single piece of float glass, instead of passing on the complexity to the optical fabricator to make an aspheric surface. The bent-mirror solution may not work in all cases, especially those involving actively-cooled mirrors. The trend in optical design for higherbrightness source instrumentation appears to be away from aspheric optics and towards spherical optics used at grazing incidence. The consensus is that spherical surfaces can be made more easily to meet the strict figure tolerances required by newer SR sources than can aspheric surfaces. However, careful consideration must be given to choice of substrate material in order not to compromise the scattered light performance of the system.

\section{Surface Figure}

Although we were successful in employing the microinterferometer in measuring the roughness of smooth surfaces, there still remains the problem of measuring the overall shape, or figure, of cylindrical aspheres. In order to utilize the small source size (vertical height $<150 \mu \mathrm{m})$ and take advantage of the inherent high brightness of the NSLS sources, the image formation properties of the mirrors need improvement. As no commercial instrumentation is available to make figure measurements on cylindrical aspheres, we 
embarked on a development program to produce an instrument that would allow us to measure the figure on cylinders up to one meter in length. Since we already had a quick and reliable method for measuring microroughness with scale lengths ranging from several micrometers up to several millimeters, we decided to build a separate instrument to cover the complementary spatial period range, from $1 \mathrm{~mm}$ up to $1 \mathrm{~m}$, rather than try to build a new instrument that encompassed the entire spatial frequency range. Our approach to cylindrical figure measurement was to employ a pencil beam interferometer [11] to make profile measurements down the length of the mirror in a non-contact manner and use the same techniques to analyze the figure data as we use to analyze the finish data [5].

The power spectral density of the surface profile has proved to be a very useful analytic tool for understanding the nature of polished surfaces and for predicting the performance of grazing incidence optics. The Fourier spectrum of the axial profile of cylindrical mirrors over the composite spatial frequency range of the two complementary profiling instruments can be used to predict the complete imaging and scattering performance of the component [12] and can be used as a specification to the manufacturer for the production of the desired surface. It serves as the point of commonality between the functional performance of grazing incidence systems at $x$-ray wavelength and the metrology made at visible wavelengths in the laboratory. The power spectral density approach to surface specification is not widely used, mainly because it is an unfamiliar concept to most traditional fabricators and because commercial interferometer analysis software does not support the calculation. One finds it used in specialized projects involving the development of sophisticated optical systems, slich as $\mathrm{x}$-ray telescope projects [13], but with the new measurement techniques now available, it should become a more common specification technique. 


\section{Surface Figure Measurement}

The remainder of this paper will be concerned with extending the range of surface profile measurements to $1 \mathrm{~m}$ distances. The instrument that we have developed in our laboratory to achieve that goal is the Long Trace Profiler (LTP). This instrument has been described in detail elsewhere $[14,15]$, so only a brief discussion of its operation is in order now. The LTP consists of an optical head mounted on an air bearing slide driven by a stepping motor lead screw drive with a free travel range of almost one meter. The optical head consists of a HeNe laser source, beamsplitting optics to produce two collinear but otherwise unaltered probe beams, and a lens and linear array detector assembly to record the interference pattern produced by the two beams after reflection from the test surface. Data collection and analysis are controlled by an HP320 computer system.

The LTP is a slope measuring interferometer. The measured parameter is the local axial surface slope between the two adjacent regions of the surface illuminated by the probe beams. The optical head is stepped along the surface in increments of one laser beam diameter $(1 \mathrm{~mm})$, and the measured slope values form the pixel function, which is surface slope in units of detector pixel number. The pixel function is converted into true slope by application of the calibration factor, which is $10.1 \mu \mathrm{rads}$ per pixel. The slope function is then converted into height by a Fourier filtering technique $[11,15]$, and the power spectral density function can be generated through further processing.

An important design criterion of the LTP is that it be able to measure absolute radius of curvature. This is accomplished by monitoring the pitch angle of the optical head in real time as it moves along the air bearing rail. We use a sensitive electronic autocollimator to measure the change in angle of the optical head as a function of position along the rail. By subtracting the optical head pitch angle from the raw slope function, we effectively 
remove all slope errors introduced by static and dynamic deflection of the air bearing beam. We do not need to rely on a precisely-calibrated special reference flat or straightedge in order to make absolute surface radius measurements: the nature of the LTP system is self calibrating.

\section{Typical Figure Measurements}

A large number of mirrors have been measured on the LTP over the past year. Most have been cylindrical aspheres, either in the shape of a partial right cylinder with a minor radius on the order of tens of millinieters (see Fig. 8), or in the shape of a toroid with a major radius on the order of kilometers or hundreds of meters. Some cylinders have been designed to be bent in the axial direction to approximate a toroid. The LTP has proven to be an extremely useful device for analyzing the performance of mirror bending mechanisms, allowing one to compare the actual shape of the bent mirror to the predicted shape as a function of applied load. Most cylinder mirrors that have been measured on the LTP suffer from common manufacturing defects. It is a useful exercise to review these problems in order to make them known to potential users of cylindrical aspheres and to feed back the metrology information to the manufacturer. The goal of this exercise is to stimulate improved production techniques for aspheric optics.

A schematic diagram of a typical grazing incidence cylinder mirror is shown in Fig. 8. Most cylinders in use at the NSLS are 60 to $70 \mathrm{~cm}$ in length. Most suffer from figure errors in common with the following example. The raw slope function measured on a diamond-machined and polished single crystal silicon mirror is shown in Fig. 9. Several features of interest are evident in this plot. The overall tilt of the curve from upper left to lower right indicates that this surface has a large non-zero curvature. (The derivative of an 
ideal surface with curvature is a sloping straight line.) Something is wrong at each end of the mirror because the slope function departs from the average tilt and becomes steeper at each end. And most obvious is a uniform periodicity in the center segment with an amplitude of several microradians and a period of exactly $20 \mathrm{~mm}$.

The surface height profile computed from the raw slope function is shown in Fig. 10. One can see immediately that the non-zero slope is the result of a large convex curvature in the surface with a sag of about $20 \mu \mathrm{m}$. Such a surface will not function well as a focusing element! Our analysis software allows us to remove the tilt from the slope function over the central region of the surface, which is equivaient to removing the curvature from the height profile. If we perform these steps and calculate the height from the detrended slope function, we see the residual surface profile in Fig. 11. This curve is the departure of the surface from the radius best-fit to the central $500 \mathrm{~mm}$ of the surface. One notes immediately that the steep slope regions near each end are a result of edge roll-off in the finished surface. Presumably the quality of the diamond-machined surface was compromised by the polishing techniques as evidenced in the edge roll-off region and in the overall convex curvature along the axis. Convex curvature and edge roll-off are common problems seen in most cylindrical surfaces in varying degrees. This is a rather extreme example of overall curvature but is a typical example of edge roll-off. The most unusual aspect of this surface does not show up well in the height profiles, except at higher magnification of the vertical scale, but is readily apparent in the slope function plots, that being the $20 \mathrm{~mm}$ periodicity in the central region of the surface. If we compute the power spectrum of the residual height profile of Fig. 11 and plot the result in Fig. 12, we can see the signature of the $20 \mathrm{~mm}$ periodicity standing out from the continuum background as a sharp spectral line. The area under the spectral line implies that the surface has an RMS 
roughness of $22 \AA$ at the $20 \mathrm{~mm}$ period, which is equivalent to a $20 \mathrm{~mm}$ period sinusoid with a peak-to-valley amplitude of $63 \AA$ extending over the length of the surface. The maximum slope error produced by this idealized sinusoid is $\pm 1 \mu \mathrm{rad}$, or about 0.2 arcseconds. This happens to be woll within the specifications for this mirror surface, which allow several arcseconds of error at these spatial frequencies. If the $20 \mathrm{~mm}$ periodicity were the only error source on this mirror, it would be acceptable. However, the $20 \mu \mathrm{m}$ convex sag renders the mirror unusable, even though it is intended to be used in a bending apparatus. The bender is designed to produce a concave sag of about $17 \mu \mathrm{m}$. If this mirror were installed, the bender would first have to unbend the $20 \mu \mathrm{m}$ convex curvature, which would require large forces outside the desired range of operation. This mirror was returned to the marufacturer for additional figuring.

Also shown in Fig. 12 at the higher spatial frequencies (greater than $2 \times 10^{-4}$ ) is the power spectrum of the silicon mirror surface measured with a WYKO NCP 1000 Digital Optical Profiler with a $2.5 \mathrm{X}$ objective. This is a result our standard measurement method and analysis technique to quantify the roughness characteristics of surfaces over the $5 \mathrm{~mm}$ to $10 \mu \mathrm{m}$ spatial period range. The WYKO-range spectrum is an average over several spectra calculated from profile measurements made at random locations along the length of the cylindrical surface. The most outstanding feature of the composite spectrum is the smooth transition between the two measurement techniques: the magnitude of the two spectra in the region of overlap is the same, although the profiles were measured by two completely different techniques, and the slope of the composite spectrum is constant over an enormous range of spatial frequencies, corresponding to periods from $100 \mathrm{~mm}$ down to $10 \mu \mathrm{m}$. The composite spectrum has a $1 / f^{\mathbb{a}}$ power law shape with a spectral index of about $\mathrm{n}=-\mathbf{1 . 3 3}$ 
The surface spectrum can be used to compute the RMS roughness statistics of the surface by integrating the area under the curve over selected bandwidth regions. If we integrate over the WYKO region from $5 \mathrm{~mm}$ down to $10 \mu \mathrm{m}$, we get an RMS roughness value of $10.95 \AA$, which is a reasonably good finish for this type of mirror. The corresponding RMS roughness number for the LTP region over the $100 \mathrm{~mm}$ to $4 \mathrm{~mm}$ bandwidth is $57.5 \AA$, which includes the $22 \AA$ contribution from the spectral line at $20 \mathrm{~mm}$. The high frequency cutoff, e.g. Nyquist frequency, for the LTP data occurs at the $4 \mathrm{~mm}$ period because the sampling interval in this data set is $2 \mathrm{~mm}$ per data point. The large amplitude of the LTP spectrum at the longest spatial periods, between $500 \mathrm{~mm}$ and $100 \mathrm{~mm}$, results in an RMS roughness value of $934 \AA$. The source of this large low frequency component is readily apparent in the residual height profile as the height difference between the left and right halves of the mirror. By eye, the peak-to-valley height difference appears to be somewhat less than $1 \mu \mathrm{m}$, which is in agreement with the computed RMS value of nearly $0.1 \mu \mathrm{m}$. Surface profile errors in this spatial period range are usually classified as figure errors and are usually expressed in units of waves or wavelengths of light. But there is usually some ambiguity in the meaning expressed in those terms as to whether the error refers to wavefront error or actual surface error. With the RMS error expressed in real distance units ( $\mu \mathrm{m}, \mathrm{nm}$, or Ångstroms), there is no ambiguity in the meaning of residual surface height error.

The LTP offers a rapid and accurate means to measure the performance of mirror bending apparatus. A common technique for producing axial curvature in a cylindrical or plane surface is to bend it with appropriately-placed force couples near each end of the mirror. Often the performance of the bending mechanism does not follow its design criteria and a period of debugging and modification is necessary to understand the behavior of the 
mechanism. The LTP allows one to assess the performance of the bending system off-line in a controlled laboratory environment. Examples of profile measurements on mirrors installed in two different bending mechanisms are shown in Figs. 13 and 14. Measurements on a thin fused silica cylinder bent into the shape of a toroid are shown in Fig. 13. The thin cross section (about $8 \mathrm{~mm}$ thick) is easily bent into the $100-200$ meter radius range. The total sag over the $500 \mathrm{~mm}$ clear aperture is over $300 \mu \mathrm{m}$ for the $\mathrm{R}=100 \mathrm{~m}$ case. Measurements over this sag depth range are difficult for most other optical profiling systems. The limitation on the measurement range of the LTP is not one of total sag, but rather depends on the maximum slope of the surface. In the present configuration the aperture of the Fourier transform lens limits the maximum surface slope to be \pm 5 mrads. The above example has a maximum slope of only \pm 2.5 mrads. The last example in Fig. 14 is for a single-crystal silicon mirror in a different bending mechanism. The mirror is a sister mirror to the one featured in Fig. 4 but does not exhibit the $20 \mathrm{~mm}$ periodicity error. It is, however, slightly more irregular than the first one. After a period of debugging problems with the bending mechanism, the mirror was successfully bent into the desired radii in the range from $4.9 \mathrm{~km}$ to $12.2 \mathrm{~km}$. This mirror is designed for use at extreme grazing incidence angles to focus kilovolt energy $x$-rays. The curvature on the silicon mirror is much shallower than the other mirror which is designed to focus much lower energy EUV photons at a shorter focal distance.

\section{Conclusion}

The measurement of the surface quality of large cylindrical aspheres is now a routine procedure in our laboratory. Profile measurements over the length of the mirror allow one to quickly and easily characterize the quality of the surface. The Long Trace Profiler allows 
us to measure the figure quality of large mirrors, and the WYKO profiler allows us to measure the finish. We have demonstrated that, at least in the case of the silicon mirror surface, the power spectral density function follows a power law curve over a wide range of spatial frequencies. Further work is necessary to characterize the spectra of other surfaces over the LTP range. We have also shown that certain manufacturing errors are common to cylindrical aspheres and that we have the metrology capability to provide the necessary feedback to the manufacturers so that they might improve their production techniques.

\section{References}

[1] J. C. Wyant, C. L. Koliopoulos, B. Bushan, and O.E. George, "An Optical Profilometer for Surface Characterization of Magnetic Media," ASLE Trans. 27, 101 (1984).

[2] J. M. Eastman and J. M. Zavislan, "A New Optical Surface Microprofiling ; Instrument," Proc. SPIE 429 (1983).

[3] G. E. Sommargren, “Optical Heterodyne Profilometry," Appl. Optics 20, 610 (1981).

[4] J. F. Biegen and R. A. Smythe, "High Resolution Phase Measuring Laser Interferometric Microscope for Engineering Surface Metrology," in: Scanning Microscopy Technologies and Applications, E. Clayton Teague, ed., Proc. SPIE 897, 207216 (1988).

[5] E. L. Church and P. Z. Takacs, "Use of an Optical-profiling Instrument for the Measurement of the Figure and Finish of Optical-quality Surfaces," WEAR 109, 241257 (1986). 
[6] E. L. Church, H. A. Jenkinson, and J. M. Zavada, "Relationship Between Surface Scattering and Microtopographic Features," Opt. Eng. 18125 (1979).

[7] E. L. Church and P. Z. Takacs, "The Interpretation of Glancing Incioence Scattering Measurements," Proc. SPIE 640, 126 (1986).

[8] In preparation for submittal to Applied Optics.

[9] Paul Glenn, "Space Telescope Performance Prediction Using the OSAC Code," in: Large Optics Technology, Proc. SPIE 571, 164-179 (1985).

[10] K. W. Jones, P. Z. Takacs, J. B. Hastings, J. M. Casstevens, and C. Pionke, "Fabrication of an 8:1 Ellipsoidal Mirror for a Synchrotron X-ray Microprobe," in: Metrology: Figure and Finish, Bruce Truax, ed., Proc. SPIE 749, $37-44$ (1987).

[11] K. von Bieren, "Interferometry of Wavefronts Reflected Off Conical Surfaces," Appl. Opt. 22, 2109 (1983).

[12] E. L. Church and P. Z. Takacs, "Prediction of Mirror Performance from Laboratory Measurements,"X-ray/EUV Optics for Astronomy and Microscopy, R. B. Hoover, ed., Proc. SPIE 1160, 323-336 (1989).

[13] Paul Glenn, "Space Telescope Performance Prediction Using the OSAC Code," Large Optics Technology, Proc. SPIE 571, 164-179 (1985).

[14] Peter Z. Takacs, Shi-nan Qian and Jeffrey Colbert, "Design of a Long-Trace Surface Profiler," Metrology-Figure and Finish, Bruce Truax, ed., Proc. SPIE 749, 59-64 (1987).

[15] P. Z. Takacs, S.-C. K. Feng, E. L. Church, S.-N. Qian, and W.-M. Liu, "Long Trace Profile Measurements on Cylindrical Aspheres," Advances in Fabrication and Metrology for Optics and Large Optics, Jones B. Arnold and Robert E. Parks, eds., Proc. SPIE 966, 354-364 (1989). 
TABLE I. Suppliers of Synchrotron Radiation Mirrors Included In This Survey.

American Aspheric Co.

Applied Optics Center Corp.*

Continental Optical Corp.

Diamond Electro-Optics, Inc.

Ferranti Astron, Ltd.

Frank Cooke, Inc.

General Optics

Karl Lambrecht Corporation

Liberty Mirror

Libbey-Owens-Ford

Optic-Electronic Corporation
P.C. Box 22303

Tucson, AZ 85734

Burlington, MA

15 Power Drive

Hauppauge, NY 11787

323 Andover Street

Wilmington, MA 01887

Unit 1, Aerodrome Way

Cranford Lane

Hounslow TW5 9QB

UNITED KINGDOM

59 Summer Street

North Brookfield, MA 01535

554 Finn Avenue

Moorpark, CA 93021

4204 N. Lincoln Avenue

Chicago, IL 60618

851 Third Avenue

Brackenridge, PA 15014

11545 Pagemill Road

P.O. Box 740668

Dallas, TX 75374-0668

Research Optics \& Development, Inc.* Waltham, MA

Union Carbide Nuclear Division Oak Ridge, TN

Y12 Plant

(Now under management by Martin Marietta Corporation)

*The mirror fabrication facility from this organization is now a part of Diamond Electro-Optics. 
TABLE II. Synchrotron Radiation Mirrors Measured for Surface Roughness

\begin{tabular}{|c|c|c|c|c|c|c|c|c|}
\hline \multirow{3}{*}{ Figure } & \multicolumn{4}{|c|}{ ASPHERES } & \multicolumn{4}{|c|}{ FLATS } \\
\hline & \multicolumn{4}{|c|}{ Material } & \multicolumn{4}{|c|}{ Material } \\
\hline & ENP/Al & & Glass, Ceram & & $\mathrm{ENP} / \mathrm{Al}$ & & Glass, Ceramic & \\
\hline Cylinder & $\begin{array}{l}\text { OEC } \\
\text { ROD } \\
\text { Astron }\end{array}$ & $\begin{array}{l}4 \\
1 \\
2\end{array}$ & $\begin{array}{l}\text { F. Cooke } \\
\text { Continental } \\
\text { Am. Asph. }\end{array}$ & $\begin{array}{r}24 \\
5 \\
1\end{array}$ & $\begin{array}{l}\text { OEC } \\
\text { AOC } \\
\text { ROD } \\
\text { DEO }\end{array}$ & $\begin{array}{l}3 \\
1 \\
5 \\
2\end{array}$ & $\begin{array}{l}\text { Liberty } \\
\text { F. Cooke } \\
\text { Continental } \\
\text { Continental }\end{array}$ & $\begin{array}{ll}5 & \text { (Float G) } \\
4 & \text { (FS) } \\
1 & \text { (FS) } \\
4 & \text { (SiC) }\end{array}$ \\
\hline Ellipsoid & $\begin{array}{l}\text { DEO } \\
\text { AOC } \\
\text { Unknown } \\
\text { OEC }\end{array}$ & $\begin{array}{r}18 \\
1 \\
2 \\
2\end{array}$ & $\begin{array}{l}\text { Continental } \\
\text { Unknown }\end{array}$ & $\begin{array}{l}1 \\
1\end{array}$ & $\begin{array}{l}\text { Astron } \\
\text { Y12 }\end{array}$ & $\begin{array}{l}2 \\
1\end{array}$ & $\begin{array}{l}\text { Karl L } \\
\text { Astron } \\
\text { Genl. Opt. }\end{array}$ & $\begin{array}{ll}4 & \text { (Float G) } \\
2 & \text { (SiC) } \\
4 & \text { (Si) }\end{array}$ \\
\hline Toroid & $\begin{array}{l}\text { Astron } \\
\text { AOC } \\
\text { OEC }\end{array}$ & $\begin{array}{l}1 \\
1 \\
1\end{array}$ & $\begin{array}{l}\text { Am. Asph. } \\
\text { Astron }\end{array}$ & $\begin{array}{l}1 \\
6\end{array}$ & & & & \\
\hline $\begin{array}{l}\text { Total Measurements } \\
\text { Per Category: }\end{array}$ & & 33 & & 36 & & 14 & & 24 \\
\hline
\end{tabular}




\section{Figure Captions}

Fig. 1 A typical surface profile for a smooth Au-coated SiC mirror measured with a WYKO NCP-1000 Digital Optical Profiler with a $2.5 x$ magnification objective.

Fig. 2. The power spectral density (PSD) curve for the Au-coated SiC surface, generated by averaging the PSD curves from 10 random locations on the surface. The RMS surface roughness for this surface, computed by integrating the area under the PSD curve, is $3.1 \AA$ over the full measurement width.

Fig. 3. Predicted and measured scattered light distributions for the Au-coated surface at a wavelength of $1.39 \AA$ at three angles of incidence. The measured scattered light curves (smooth lines) are normalized to the incident beam intensity: the relative intensity of the specular peak is given by the Fresnel reflectivity of the surface for each particular angle of incidence. The predicted scattered $x$-ray distributions (rough lines) computed from Eqs. (1) and (2) are overlayed on the appropriate measured curve. The agreement in the overall shape and magnitude of the Rayleigh-Rice formalism is quite good. The theory correctly predicts the asymmetry between the two sides of the scattered light distribution.

Fig. 4 Measured RMS roughness values plotted as a function of laboratory logbook volume and page number (roughly a time sequence starting in 1984 and ending in early 1988). This is not a time sequence of when each mirror was manufactured. Those marked with the left-pointing arrows were manufactured before 1984. See text for full explanation. The diagram illustrates that fabrication techniques for glass and ceramic aspheres are good enough to routinely produce surfaces with roughness values well below $10 \AA$ RMS. 
Fig. 5 Measured RMS roughness values for aspheres made from electroless nickel plate (ENP) on an aluminum substrate. The solid lines connect measurements made on the same mirror before and after repolishing. The diagram illustrates the difficulty in achieving RMS roughness values below $10 \AA$ with this material.

Fig. 6 Measured RMS roughness values for flat surfaces made from glass or ceramic materials. The solid vertical line connects measurements on a piece of float glass that shows an extreme anisotropy in surface roughness between the direction of pull (smooth) and the transverse direction (large amplitude long-period sinusoid). Most measurements lie well below the $10 \AA$ RMS level.

Fig. 7 Measured RMS roughness values for flat surfaces made from ENP/Al material. Most values lie above the $10 \AA$ level. The solid line shows the effect of a poor coating on a previously acceptable surface.

Fig. 8 Schematic of a typical $x$-ray cylinder mirror used to reflect synchrotron radiation at a grazing incidence angle of 3 mrads. Dimensions are in millimeters.

Fig. 9 Raw slope function for a $700 \mathrm{~mm}$ long single-crystal cylinder mirror measured by the LTP. Vertical axis units are in microradians. The $20 \mathrm{~mm}$ periodicity in the surface is clearly visible.

Fig. 10 Axial height profile of the single-crystal silicon mirror computed directly from the slope function in Fig. 2. The convex curvature with a sag of about $-20 \mu \mathrm{m}$ would require application of excessive force in the mirror bender to produce a concave curvature with the desired $+12 \mu \mathrm{m}$ sag.

Fig. 11 Detrended axial height profile: average radius of curvature of center section of Fig. 3 has been subtracted. Edge roll-off is clearly visible in the residual profile. The $20 \mathrm{~mm}$ periodicity is barely visible at this vertical scale. 
Fig. 12 Composite power spectrum of surface roughness for the silicon mirror. Lower right curve is an average from several WYKO profiler measurements, covering the spatial frequency range corresponding to periods between $5 \mathrm{~mm}$ and $10 \mu \mathrm{m}$. Upper left curve is from the central $500 \mathrm{~mm}$ of the Fig. 4 height profile and covers spatial periods between $500 \mathrm{~mm}$ and $4 \mathrm{~mm}$. The composite spectrum follows a power law shape with spectral index $\mathrm{n}=-1.33$ over the $100 \mathrm{~mm}$ to $10 \mu \mathrm{m}$ period range. The $20 \mathrm{~mm}$ periodicity stands out clearly above the underlying continuum and is equivalent to a $63 \AA \mathrm{P}-\mathrm{V}$ sinusoid on the surface. The large increase in PSD at the longest spatial periods arises from the step height discontinuity between the two halves of the mirror.

Fig. 13 Profiles of a thin fused silica cylinder bent into short radius toroidal shapes in a bending mechanism. The large sag illustrates the large depth of field capability of the LTP. Upper curve with small applied load has a non-circular shape, indicate a problem with the bending apparatus.

Fig. 14 Mirror bender measurements on a single-crystal silicon mirror similar to the one in the above discussion. The bending mechanism needs only produce a small amount of curvature to achieve vertical focusing at the $3 \mathrm{mrad}$ grazing incidence angle. Accurate absolute curvature measurements are possible because of realtime angular measurement head error correction from an electronic autocollimator. 


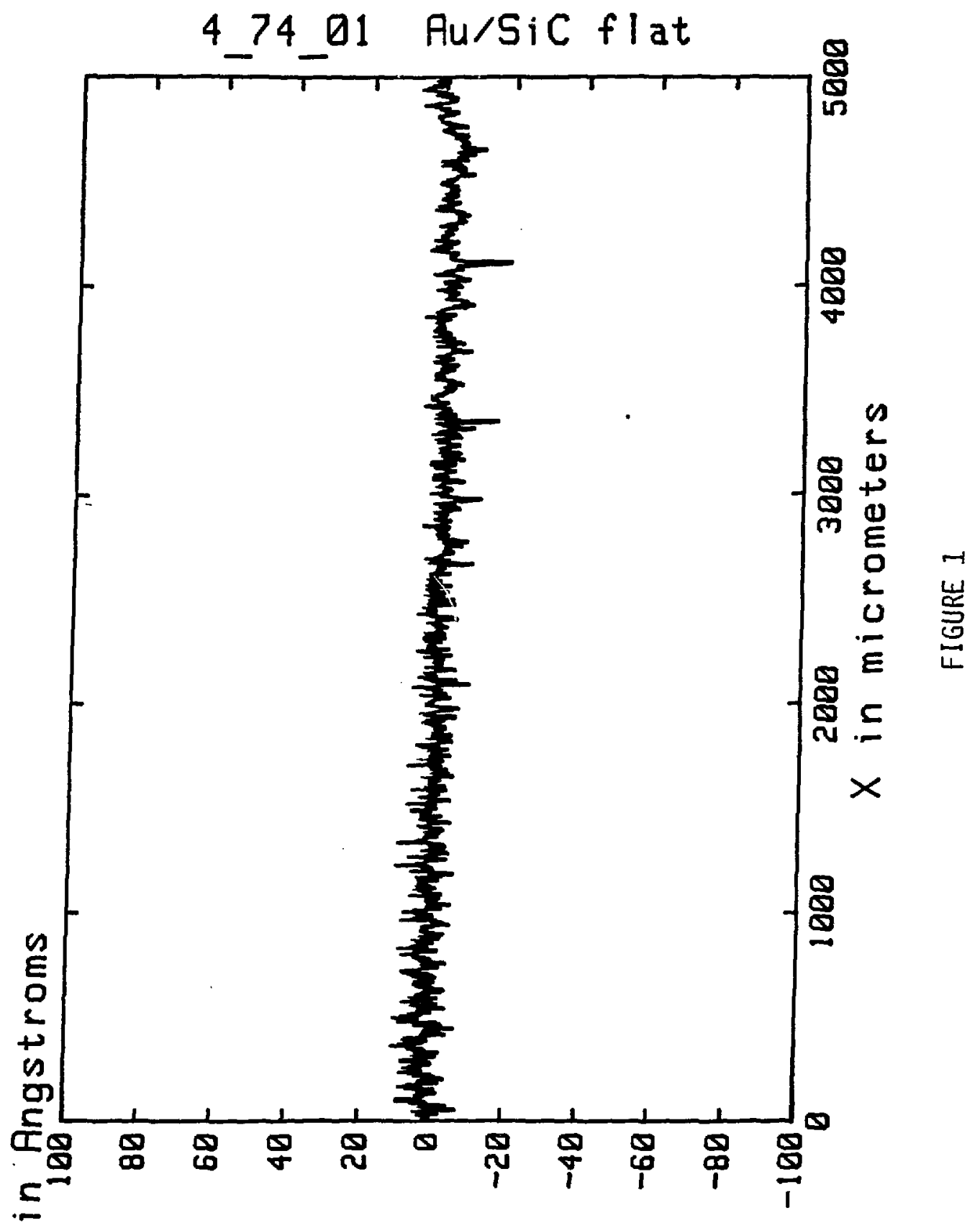

$N$ 


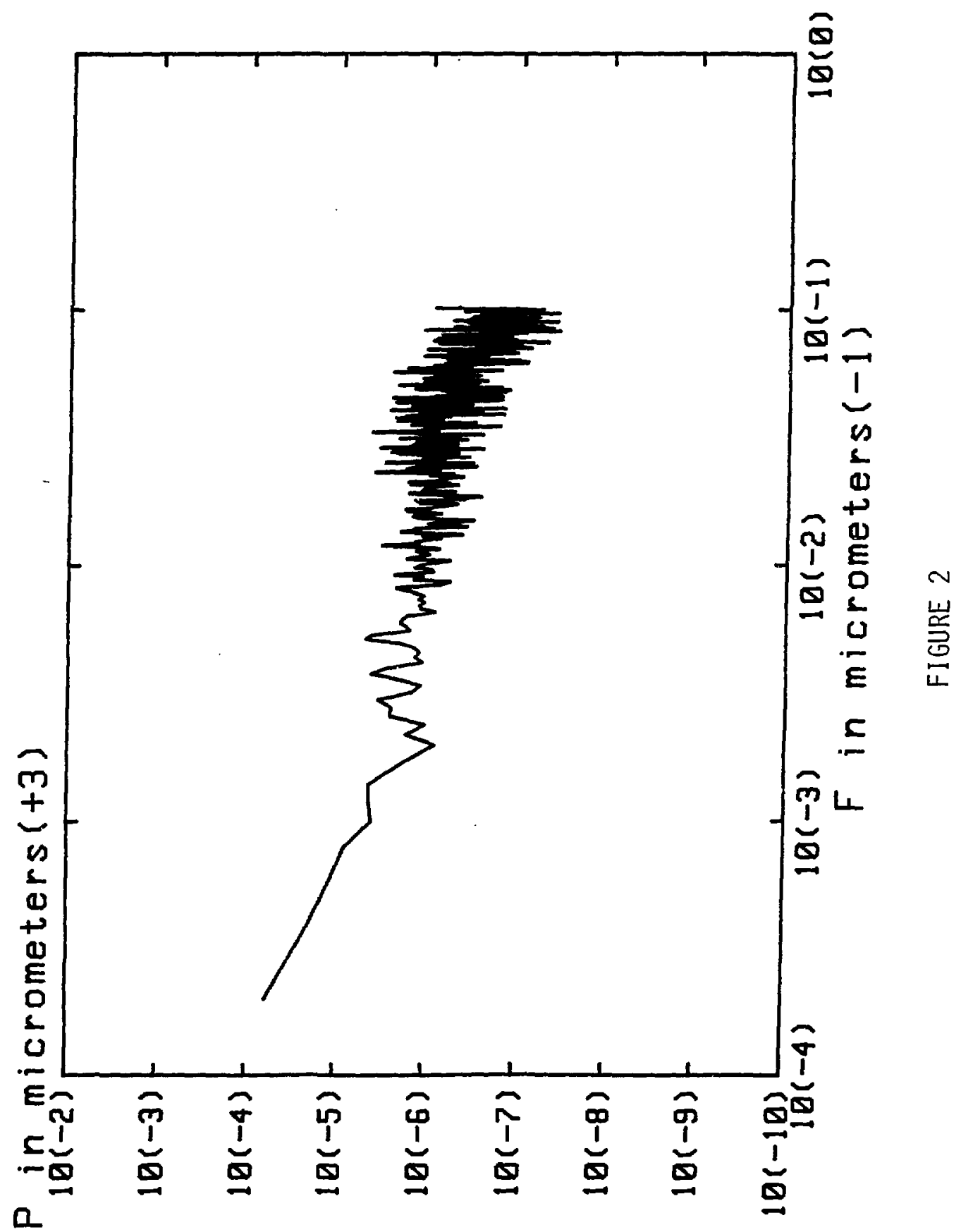




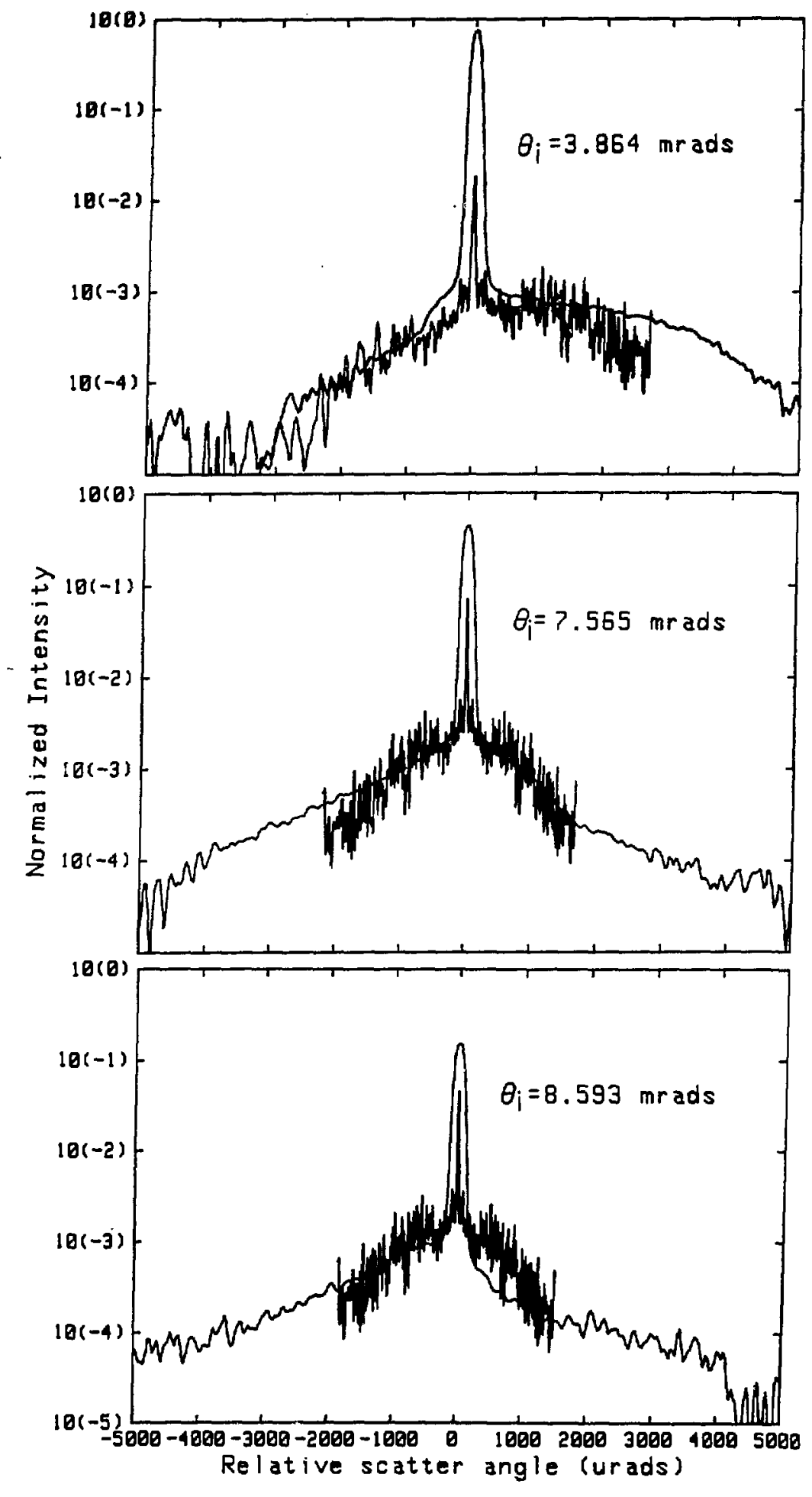

FIGURE 3 


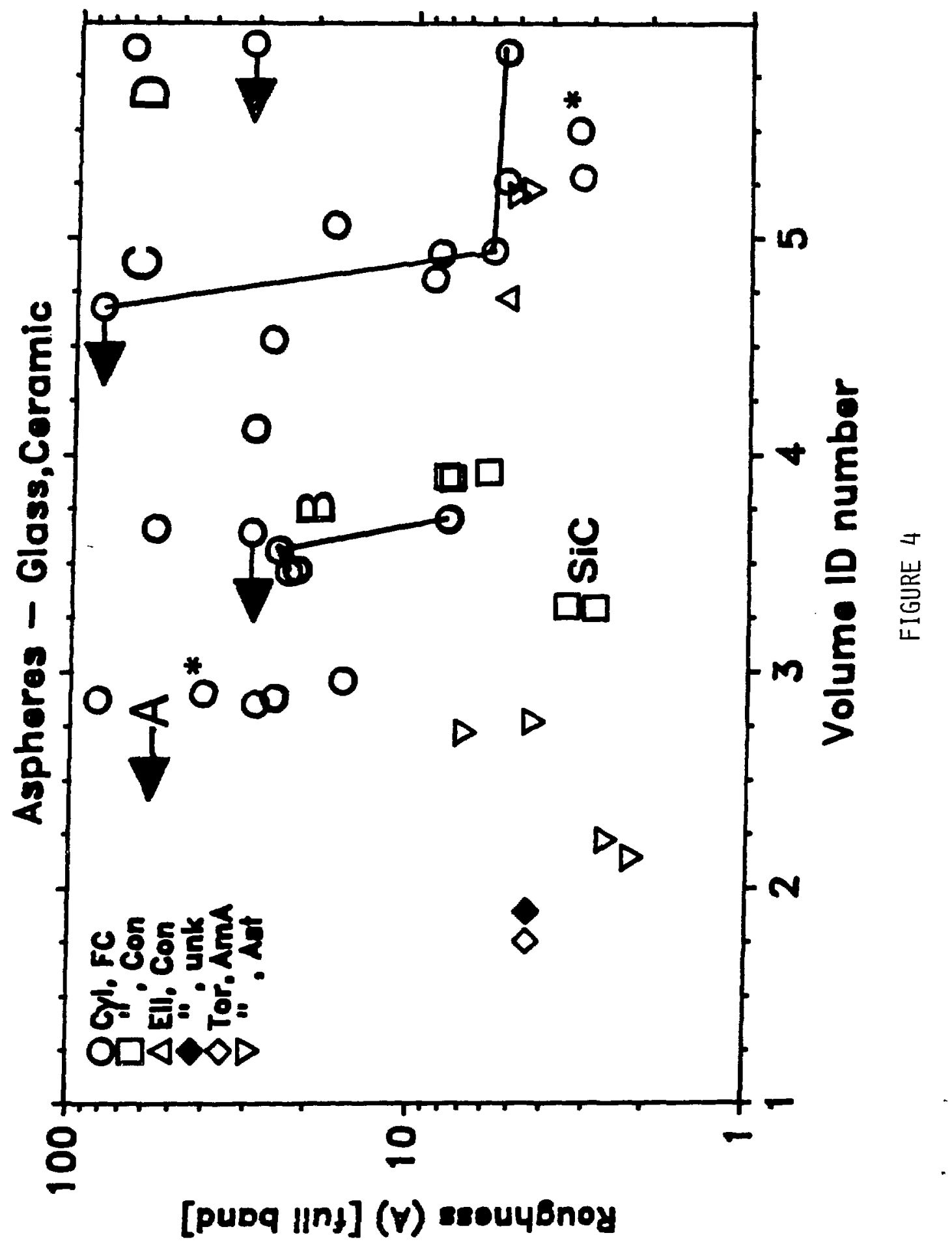




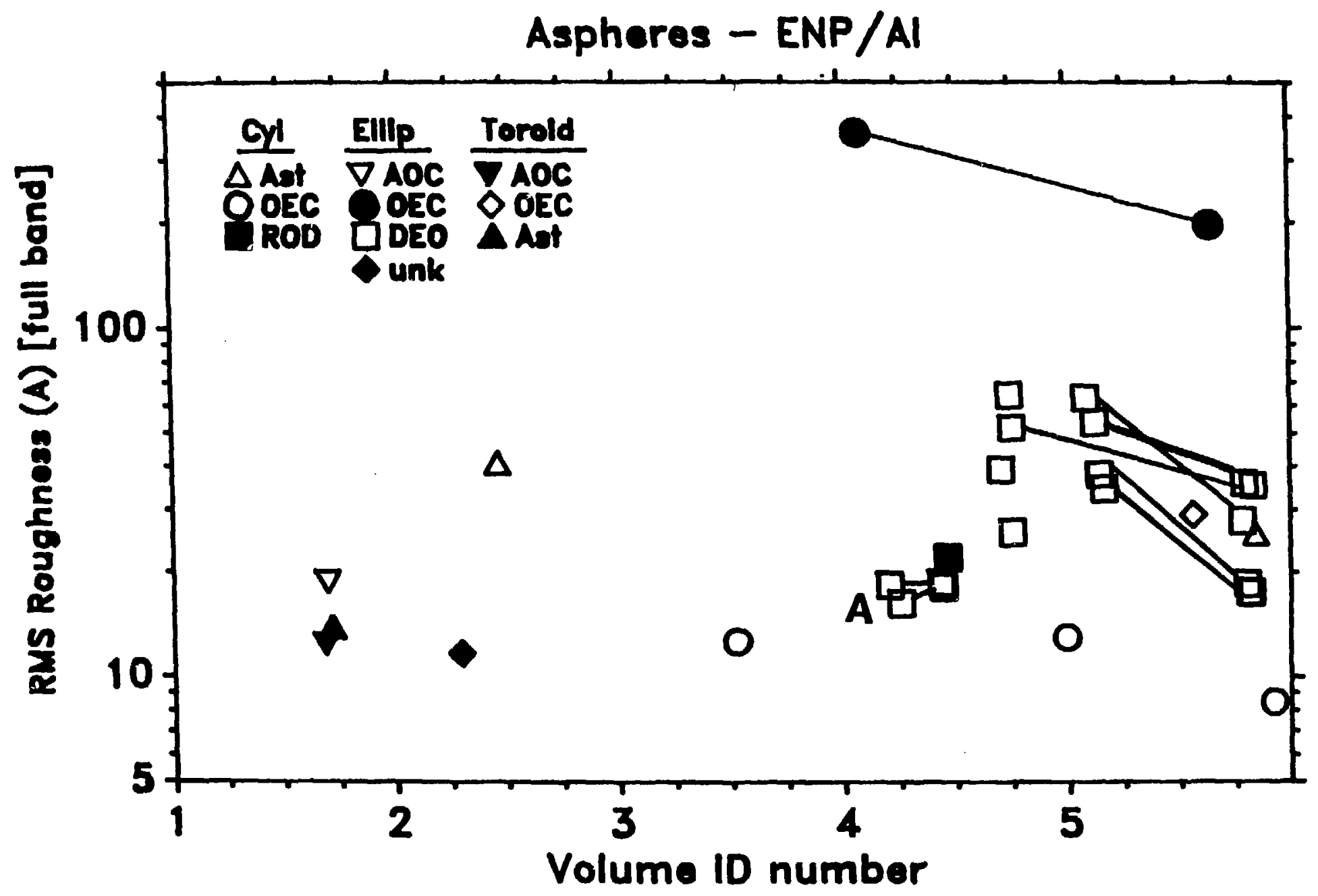

FIGLRE 5 


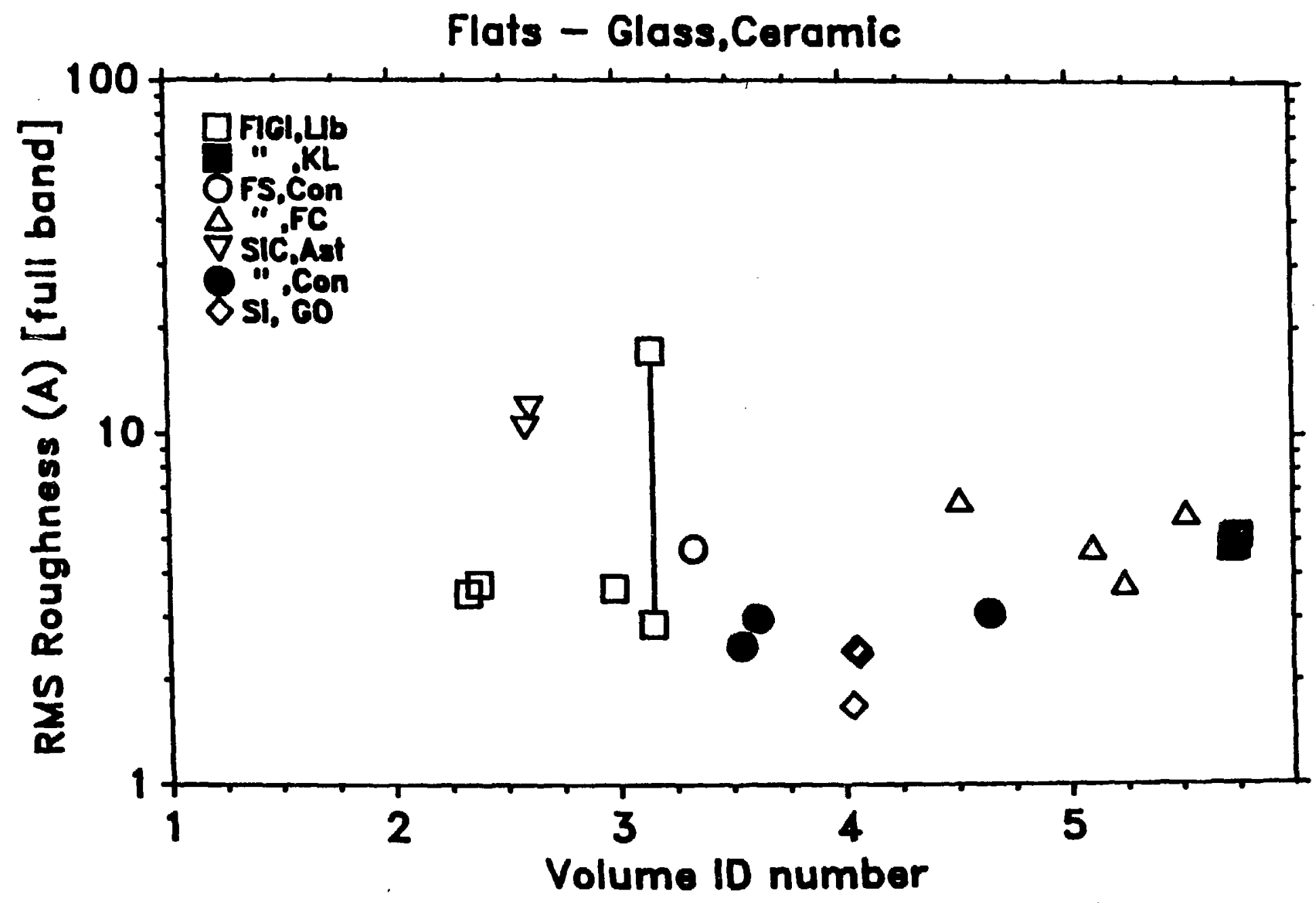

FIGURE 6 


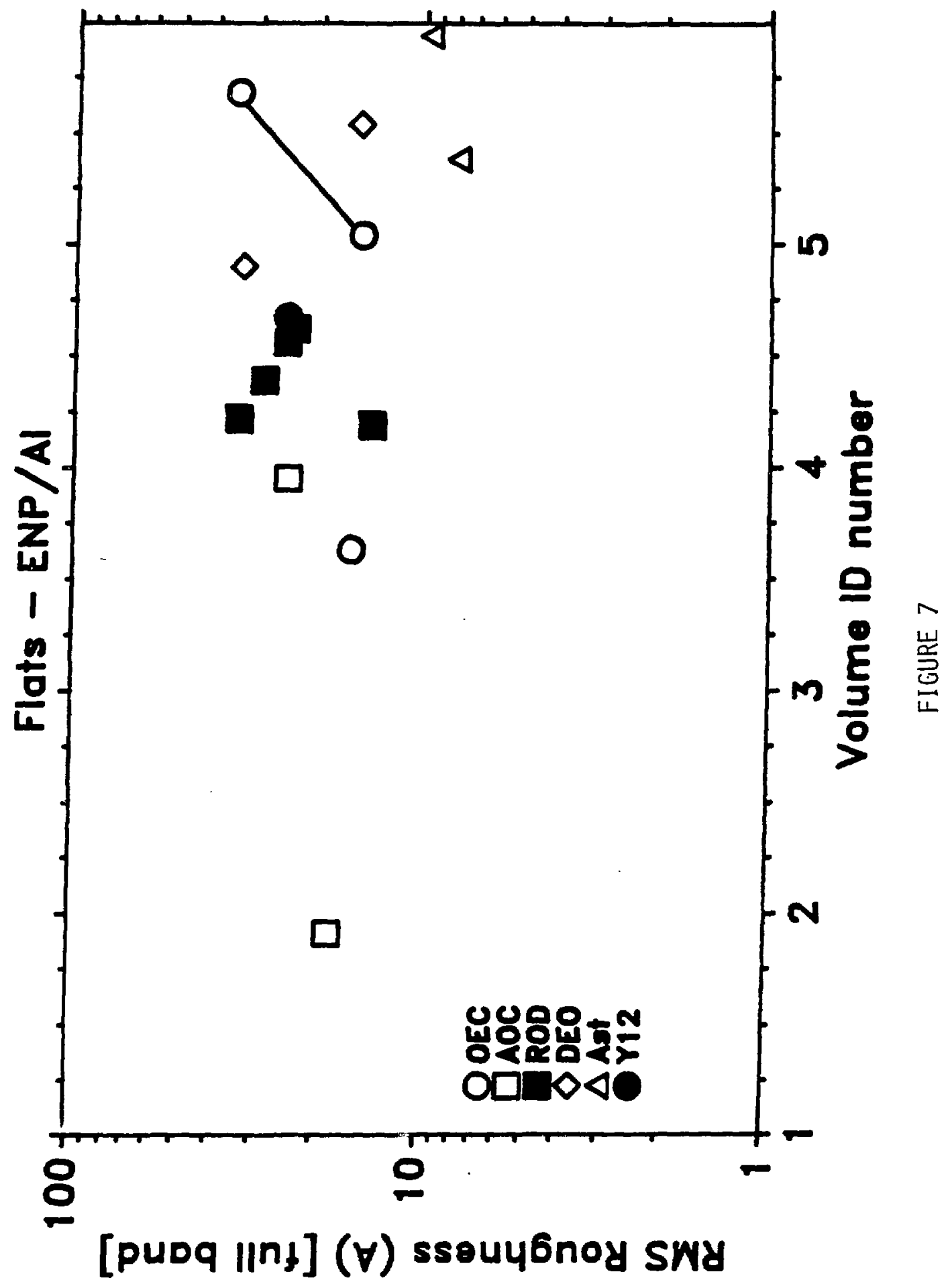




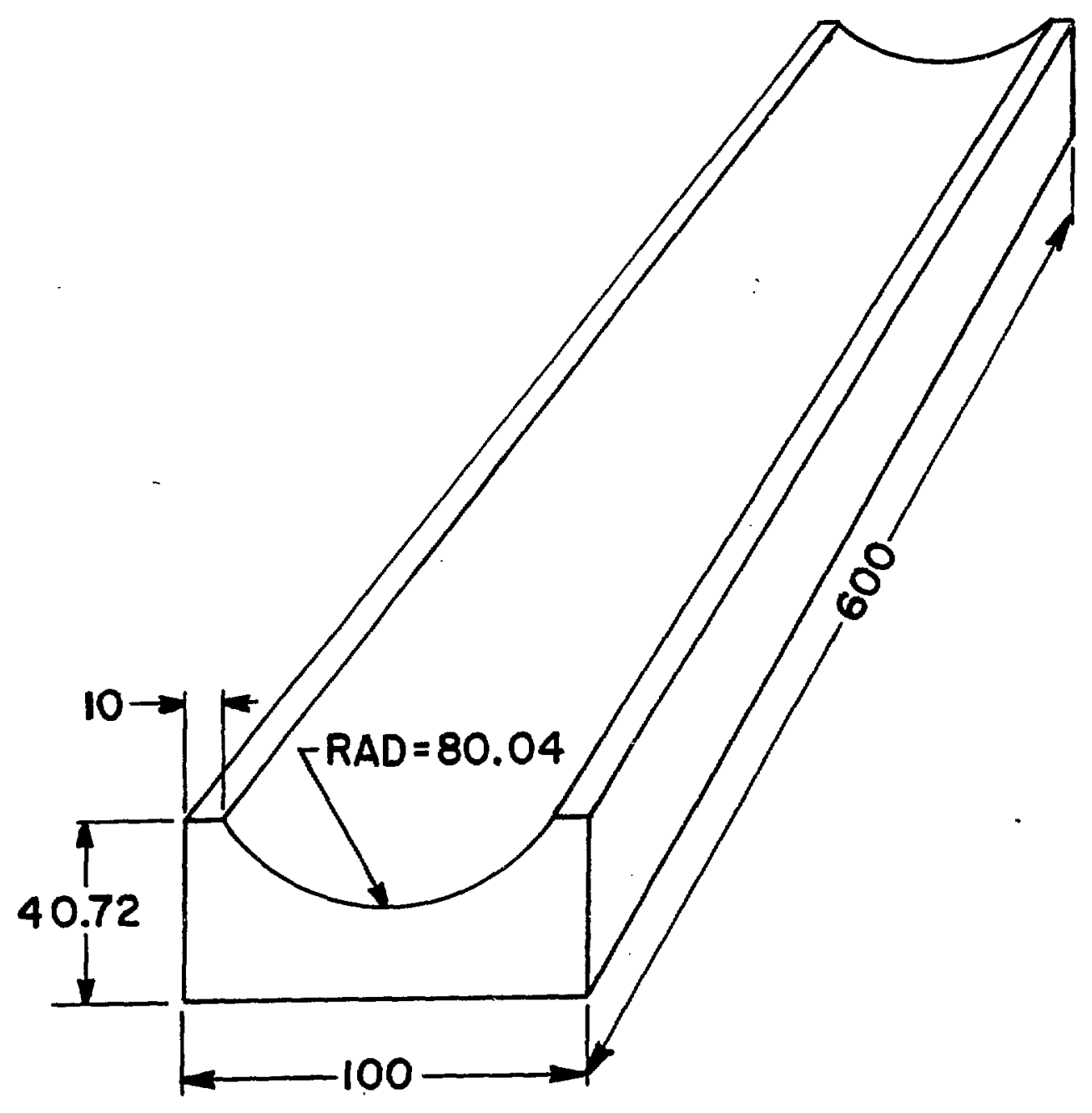

FIGURE 8 


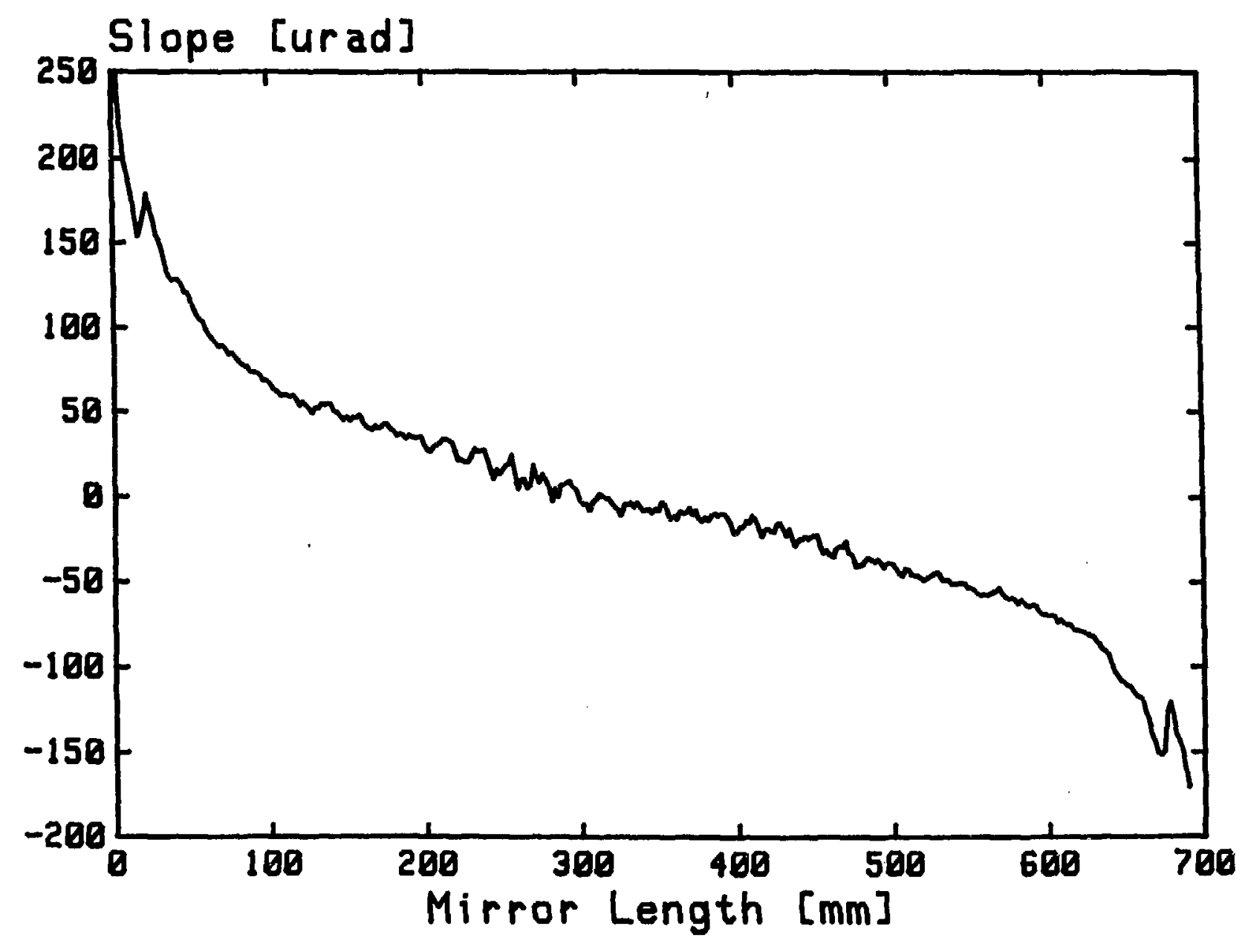

Figure 9 


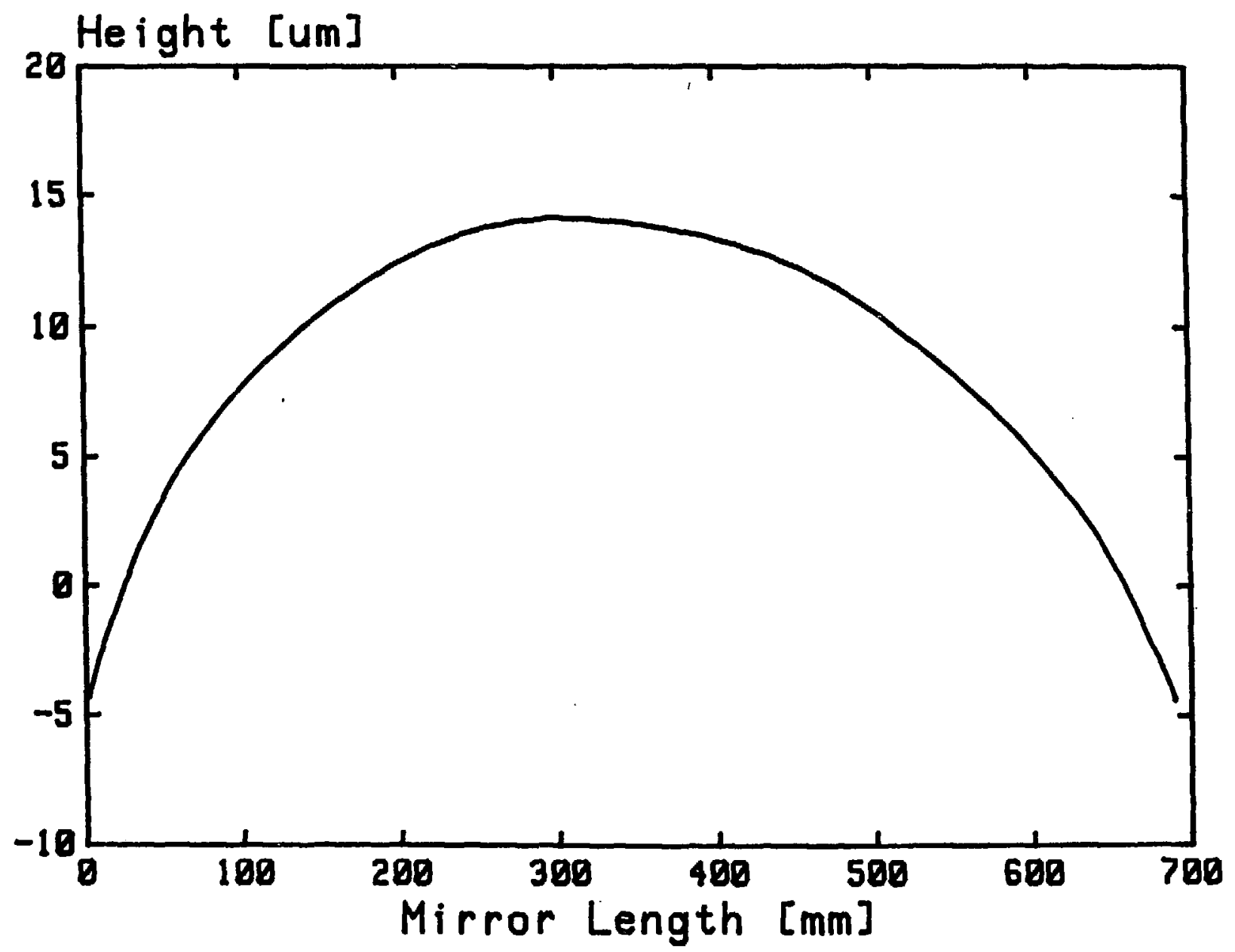

Figure 10 


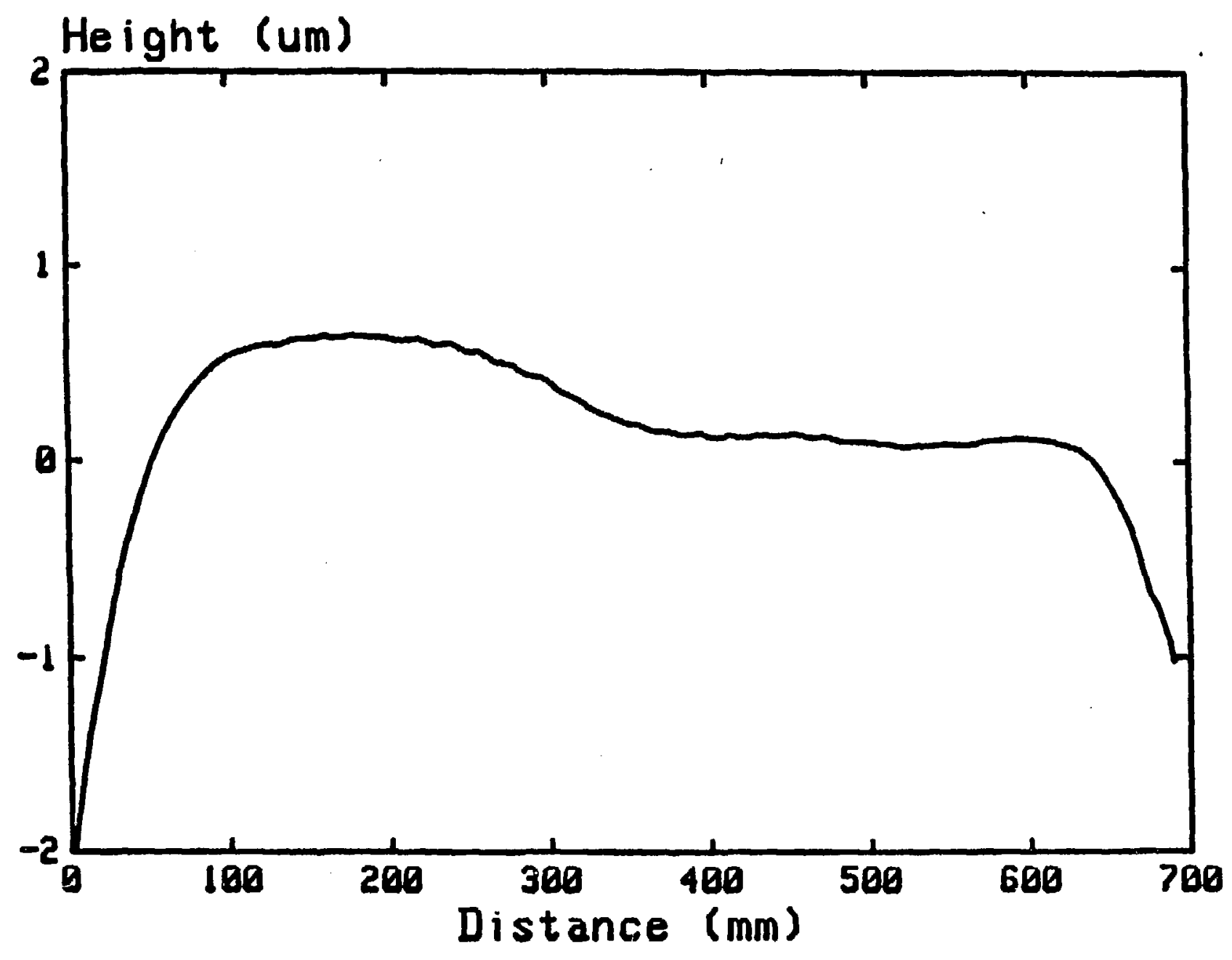

FIGURE 11 


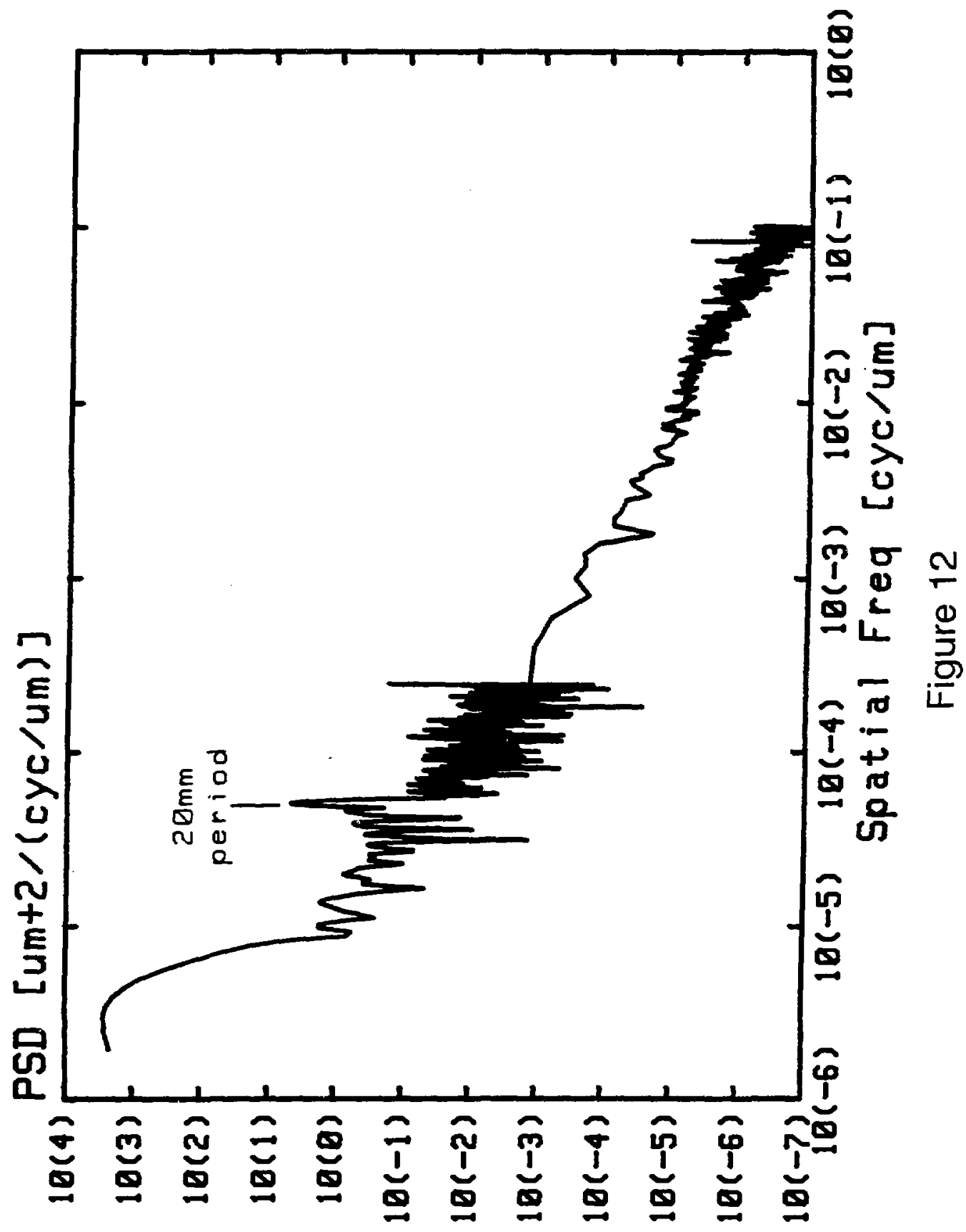




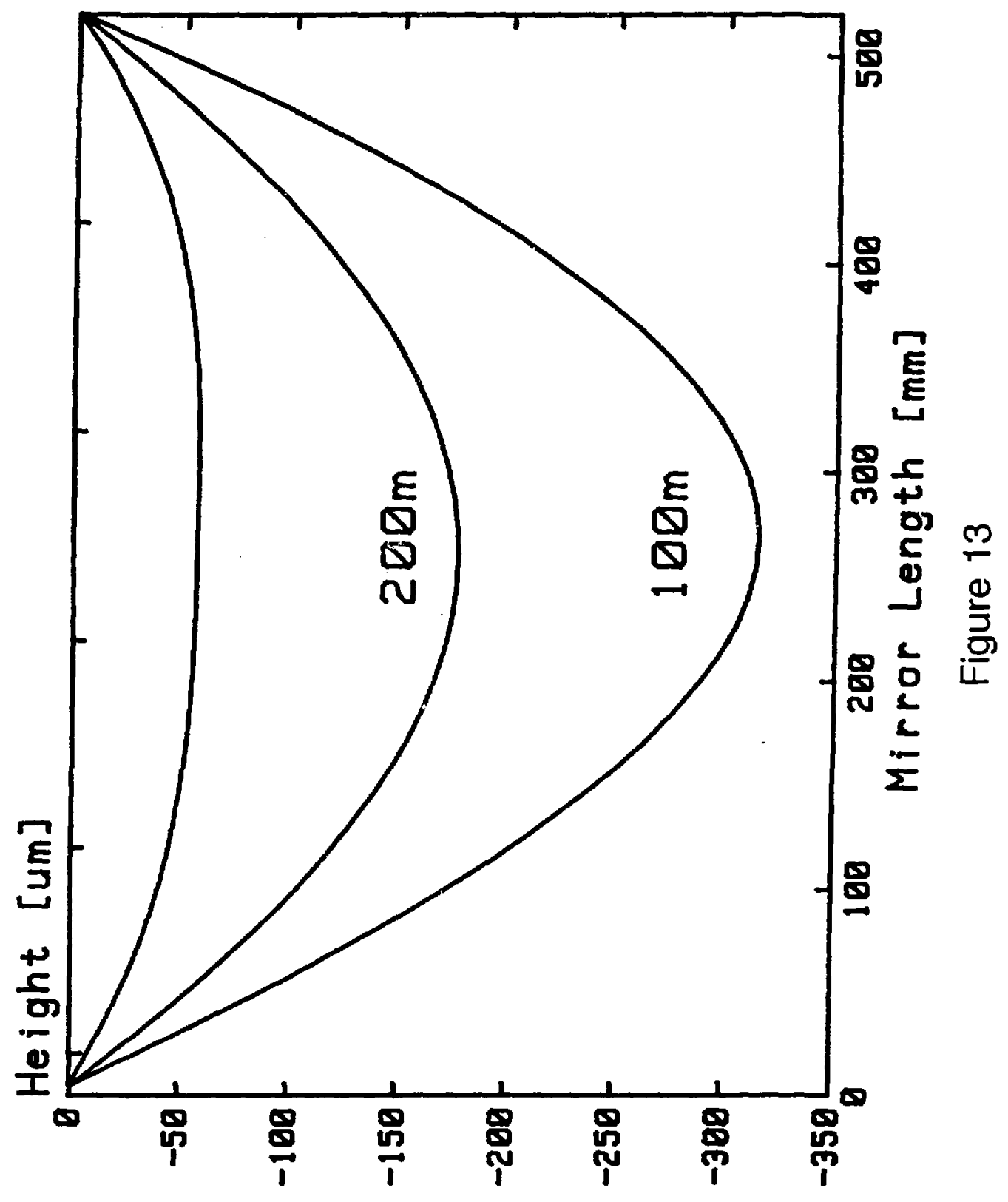




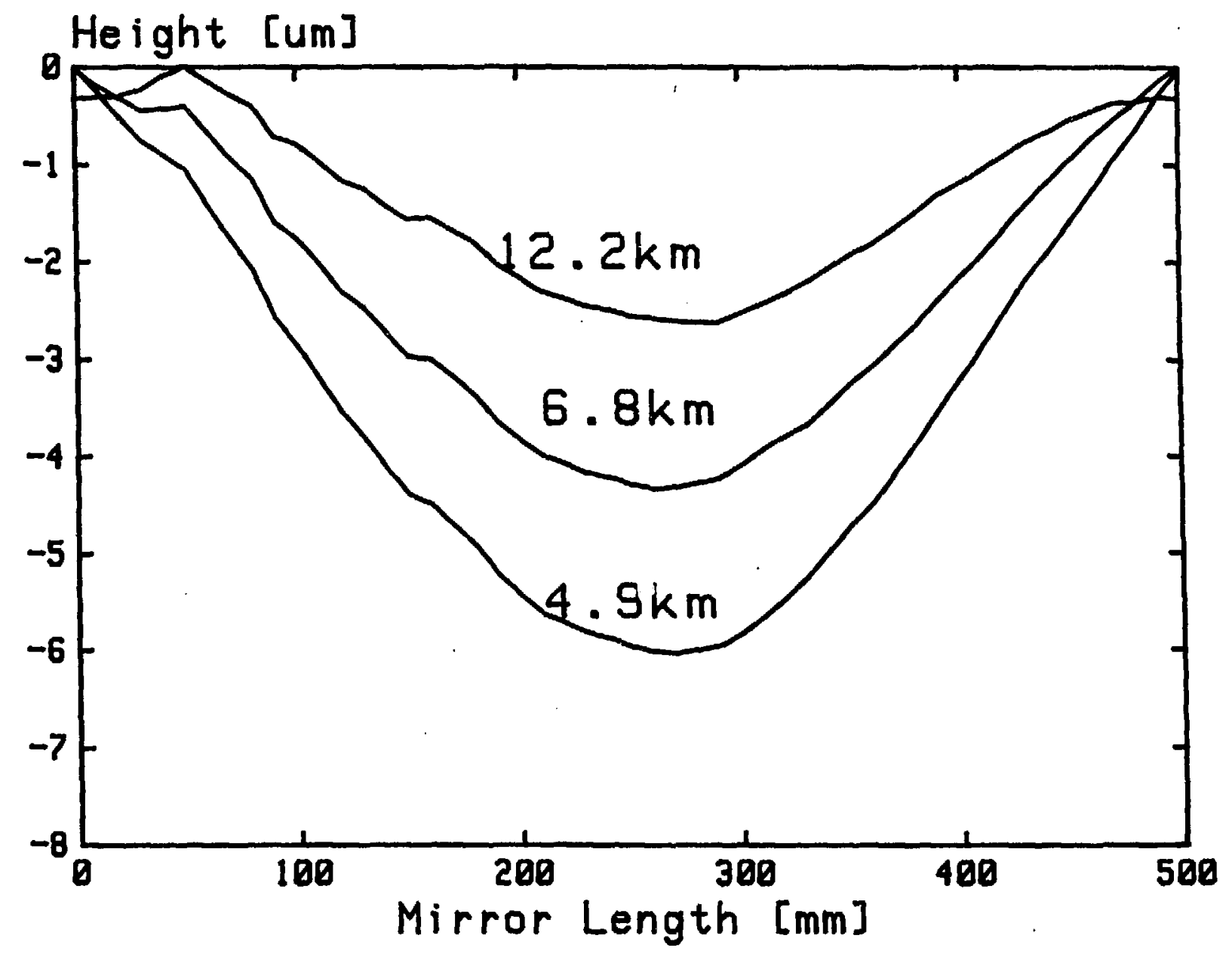

Figure 14 